\title{
Simulation and Comparison of Rheological Behavior of Branched Polymer in a Contraction-expansion Flow
}

\author{
LI Wenwen ${ }^{a}$, WANG Wei ${ }^{b^{*}}$
}

Key Laboratory of Rubber-plastics, Ministry of Education//Shandong Provincial Key Laboratory of Rubber-plastics, Qingdao University of Science \& Technology, Qingdao 266042, Shandong, China

âwenwenli0825@163.com, ${ }^{b^{*}}$ wdavid1@163.com, corresponding author.

Keywords: numerical simulation; rheology; constitutive model; contraction-expansion flow Abstract The contraction-expansion flow is a benchmark problem for exploring the rheological behavior of polymer melts by numerical method. In this study, DCPP and S-MDCPP models were adopted to describe the rheological behavior of branched polymer. The discrete elastic-viscous stress split (DEVSS), streamline-upwind (SU) techniques and the modified finite increment calculus (FIC) were employed to enhance the computational stability. The results show that the stresses predicted by S-MDCPP model agree well with the DCPP model. And the predicted the isochromatic fringe patterns of the two models have an excellent consistent with the experimental results.

\section{Introduction}

The contraction-expansion flow is not only a benchmark problem for rheologists to study the rheological behavior of polymer melts, but also common in the practical processing of polymer fluids, such as injection molding, ink-jet printer, micro-electronic device and medical application. So far, a number of literatures reported the rheological behavior of polymer melts in a contraction-expansion flow [1-4].

It is widely applicable that the rheological behavior of polymeric fluid and polymer material processing are simulated using the finite element method. Various constitutive models, which are suitable for describing the rheological behavior of branched polymer, were developed, such as POM-POM [5], XPP, DCPP [6], S-MDCPP [7]. Wang et al. [8,9] simulated the rheological behavior of polyethylene past the planar contraction and cross-slot channels, and compared with the experimental results. The numerical results agreed well with the experimental results.

In the present paper, the rheological behaviors of low-density polyethylene melts were described by the DCPP and S-MDCPP models. In order to enhance the computational stability, the modified finite increment calculus (FIC) procedure was applied to solve the velocity, pressure and stress variables using the equal low-order interpolation elements. Moreover the discrete elastic-viscous stress split (DEVSS) along with the streamline-upwind (SU) techniques were employed to deal with the convection-dominated problem.

\section{Governing Equations}

Conservation Equations. The mass and momentum conservation equations of an isothermal incompressible polymeric fluid can be expressed as (1) and (2),

$$
\begin{gathered}
\nabla \cdot \mathbf{u}=0 \\
\rho \frac{\mathrm{D} \mathbf{u}}{\mathrm{D} t}=-\nabla p+\nabla \boldsymbol{\sigma}+\rho \mathbf{g}
\end{gathered}
$$

where $\mathbf{u}$ is the velocity, $\rho$ is the fluid density, $\mathbf{g}$ is the acceleration of gravity, $p$ is the pressure, $\nabla$ denotes the gradient operator and $\boldsymbol{\sigma}$ is the extra stress tensor. $\boldsymbol{\sigma}$ includes two parts defined as (3),

$$
\boldsymbol{\sigma}=2 \eta_{v} \mathbf{D}+\boldsymbol{\tau}
$$

where $\eta_{\mathrm{v}}$ is the viscosity of the pure solvent contribution, $\mathbf{D}$ is the rate-of-deformation tensor, $\boldsymbol{\tau}$ is the viscoelastic contribution.

Constitutive Equations. The constitutive equation of DCPP model is defined as (4), (5) and (6), 


$$
\begin{aligned}
& \lambda_{\mathrm{s}} \frac{D \Lambda}{D t}-\lambda_{\mathrm{s}}(\mathbf{D}: \mathbf{S}) \Lambda+(\Lambda-1) \mathrm{e}^{2(\Lambda-1) / q}=0 \\
& \lambda_{0 \mathrm{~b}}\left[\left(1-\frac{\xi}{2}\right) \stackrel{\nabla}{\mathbf{S}}+\frac{\xi}{2} \mathbf{S}\right]+\lambda_{0 b}(1-\xi)[2 \mathbf{D}: \mathbf{S}] \mathbf{S}+\frac{1}{\Lambda^{2}}\left[\mathbf{S}-\frac{\mathbf{I}}{3}\right]=0 \\
& \tau=\frac{G}{1-\xi}\left(3 \Lambda^{2} \mathbf{S}-\mathbf{I}\right)
\end{aligned}
$$

where $\Lambda$ denotes the stretching variable, $\mathbf{S}$ denotes the orientation tensor, $\mathbf{I}$ is the identity tensor, $\tau$ is the viscoelastic contribution, $\mathrm{G}$ is the plateau modulus, and $\xi$ is the slip parameter.

The constitutive equation of the S-MDCPP model is defined as (7),

$$
{ }_{\tau}^{\nabla}+\mathbf{F}_{c}+\lambda_{0 b}^{-1} \mathbf{F}_{d}=2 G \mathbf{D}
$$

where $\mathbf{F}_{c}=\xi(\mathbf{D} \cdot \tau+\tau \cdot \mathbf{D}), \mathbf{F}_{d}=F \tau+\frac{G}{1-\xi}\left(F-e^{v(\Lambda-1)}\right) \mathbf{I} . \Lambda=\sqrt{1+\frac{1-\xi}{3 G} \operatorname{tr}(\tau)}, F=e^{v(\Lambda-1)}\left[2 r\left(1-\frac{1}{\Lambda}\right)+\frac{1}{\Lambda^{2}}\right], r=\frac{\lambda_{0 b}}{\lambda_{s}}, v=\frac{2}{q}$. $\operatorname{tr}(\tau)$ is the trace of tensor $\tau$, other parameters are the same as the DCPP model.

\section{Material Characterization and Computational Method}

In this paper, one-mode S-MDCPP model and 6-mode DCPP model were adopted to describe the rheological behavior of LDPE DOW 150R in the contraction-expansion flow. The constitutive parameters of DCPP and S-MDCPP models are shown in Table 1. The Maxwell parameters were obtained through the fitting curves of storage and loss modulus. The nonlinear parameters of DCPP model are obtained from the transient uniaxial elongational viscosity and steady-state shear viscosity.

\begin{tabular}{|c|c|c|c|c|c|}
\hline \multicolumn{6}{|c|}{ DCPP model } \\
\hline \multirow{2}{*}{$\begin{array}{l}\text { Mode } \\
\text { (i) }\end{array}$} & \multicolumn{2}{|c|}{ Maxwell parameters } & \multicolumn{3}{|c|}{ Nonlinear parameters } \\
\hline & $G_{i}[\mathrm{~Pa}]$ & $\lambda_{0 b i}[\mathrm{~s}]$ & $q_{i}=2 / v_{i}$ & $r_{i}$ & $\xi_{i}$ \\
\hline 1 & $9.4327 \mathrm{E}+4$ & $8.0651 \mathrm{E}-3$ & 2 & 1.70 & 0.1052 \\
\hline 2 & $3.8506 \mathrm{E}+4$ & $5.3023 \mathrm{E}-2$ & 2 & 1.70 & 0.0917 \\
\hline 3 & $2.1049 \mathrm{E}+4$ & $3.4859 \mathrm{E}-1$ & 3 & 1.70 & 0.1955 \\
\hline 4 & $1.1142 \mathrm{E}+4$ & $2.2918 \mathrm{E}+0$ & 3 & 1.59 & 0.0825 \\
\hline 5 & $5.1137 \mathrm{E}+3$ & $1.5067 \mathrm{E}+1$ & 3 & 1.73 & 0.0001 \\
\hline 6 & $1.7670 \mathrm{E}+3$ & $9.9056 \mathrm{E}+1$ & 2 & 4.64 & 0.0896 \\
\hline \multicolumn{6}{|c|}{ S-MDCPP model } \\
\hline \multirow{2}{*}{ Mode } & \multicolumn{2}{|c|}{ Maxwell parameters } & \multicolumn{3}{|c|}{ Nonlinear parameters } \\
\hline & $G[\mathrm{~Pa}]$ & $\lambda_{O b}[\mathrm{~s}]$ & $q=2 / v$ & $r$ & $\xi$ \\
\hline 1 & $1.3213 \mathrm{E}+4$ & 20.5094 & 2 & 3 & 0.01 \\
\hline
\end{tabular}
The fitting curves are shown in Figs. 1 (a)-(c). For the S-MDCPP model, the nonlinear parameters are obtained through the steady-state shear viscosity, as shown in Fig. 1 (a).

Table 1 Constitutive parameters of DCPP and S-MDCPP models $\left(\mathrm{T}=160^{\circ} \mathrm{C}\right)$

The contraction-expansion flow of LDPE melt described by the DCPP model was calculated by Polyflow software. The discrete elastic-viscous stress split (DEVSS) and streamline-upwind (SU) techniques were applied to solve the convection-dominated problem, in which the quadratic interpolation for velocity, linear interpolations for stress and pressure were adopted in Polyflow. When the contraction-expansion flow was simulated and the LDPE melt was characterized by the S-MDCPP model, the modified finite increment calculus (FIC) procedure was employed to reformulate the mass conservation equation for utilizing the velocity-pressure-stress equal low-order interpolation elements. 


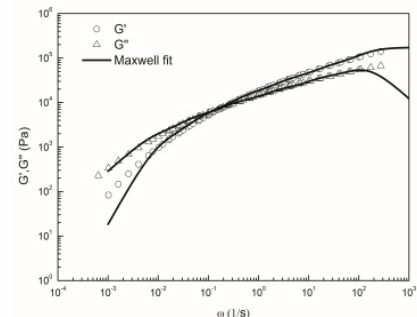

(a)

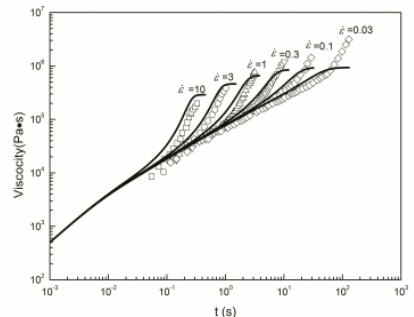

(b)

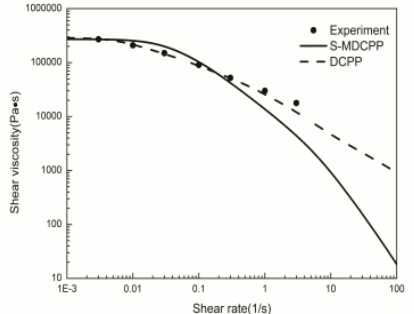

(c)

Fig. 1. (a) Storage and loss modulus fitted curve of Maxwell model. (b) Transient uniaxial elongational viscosity fitted curve of DCPP model. (c) Fitting curves of steady-state shear viscosity of DCPP and S-MDCPP models

\section{Planar Contraction-expansion Flow}

Fig. 2 shows the schematic of a 7:1:7 planar contraction-expansion channel. In order to obtain the fully developed flow, the upstream and downstream channels are long enough. Due to the symmetry of geometry and boundary conditions, only half of the contraction-expansion channel is taken. The no-slip boundary conditions are applied on the wall. The pressure of the outlet at the centerline is set to zero. The computational mesh is shown in Fig. 3.

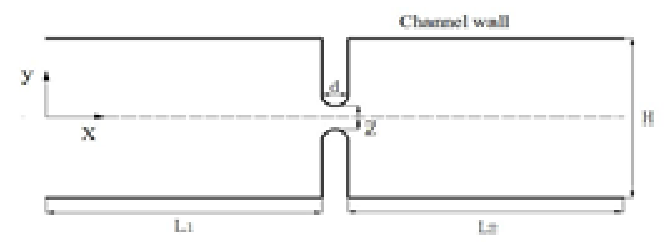

Fig. 2. Schematic of planar contraction-expansion flow

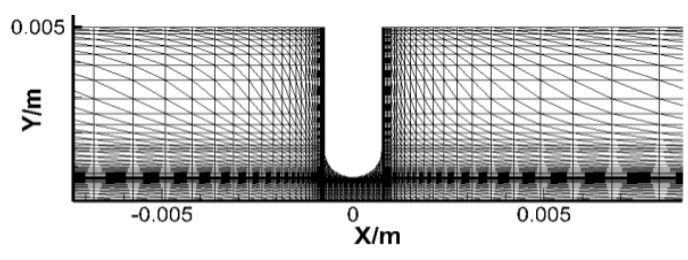

Fig. 3. Computational mesh

\section{Results and discussion}

Comparisions of stress. Figs. 4 (a)-(c) show the profiles of principle stress difference, stress $\tau_{\mathrm{xx}}$ and the first normal stress difference along the centerline predicted by the S-MDCPP and DCPP models, respectively. The principle stress difference predicted by S-MDCPP model agrees well with that predicted by DCPP model, and is consistent with the experimental results reported by Hassell [3]. The stress $\tau_{\mathrm{xx}}$ has a good agreement for the two models. In the contraction inlet, the velocity of the fluid increases quickly because of the channel getting narrow, which will induce the macromolecule chains to stretch strongly, so the stress increases rapidly. The normal stress differences predicted by the two models have a good agreement.

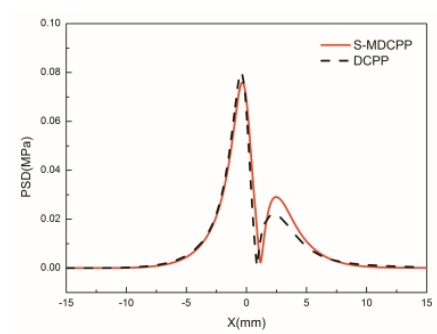

(a)

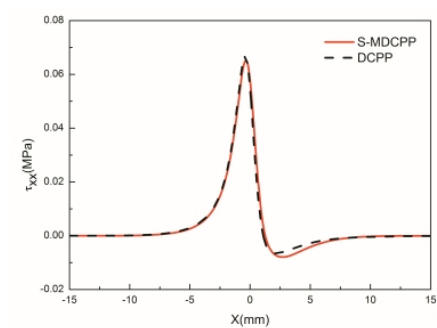

(b)

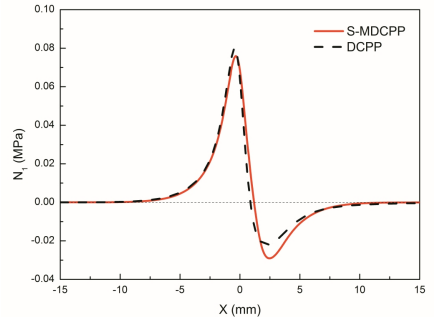

(c)

Fig. 4. (a) Profiles of principle stress difference, (b) Profiles of stress $\tau_{\mathrm{xx}}$ and (c) Profiles of normal stress difference along the centerline of the contract-expansion channel predicted by S-MDCPP and DCPP models

Comparisons of isochromatic fringe pattern. The comparisons of isochromatic fringe patterns between experimental and numerical results of the S-MDCPP and DCPP models are given in Fig. 5 and Fig. 6, respectively. The results predicted by the two models agree well with the experiments of Hassell [4]. Therefore, both constitutive models can well describe the rheological behavior of branched polymer melts. 


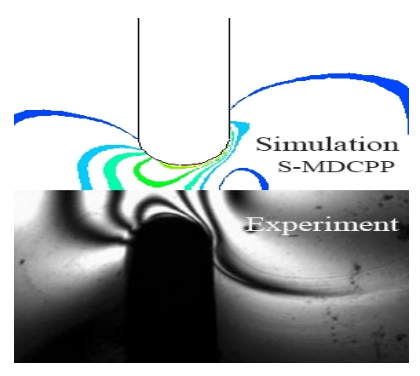

Fig. 5. Comparison of the isochromatic fringe patterns between experimental and numerical results of S-MDCPP model

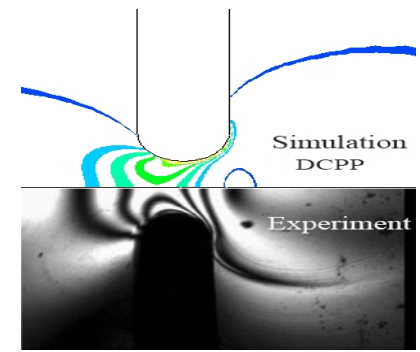

Fig. 6. Comparison of the isochromatic fringe patterns between experimental and numerical results of DCPP model

\section{Conclusions}

The fitting curves and the isochromatic fringe patterns predicted by the S-MDCPP and DCPP models agreed well with the experimental results. Hence the two models can capture and reproduce the complex rheological behavior of branched polymer.

\section{Acknowledgements}

The authors gratefully acknowledge the financial support from the National Natural Science Foundation of China through Contract/Grant number 21274072.

\section{References}

[1] J.E. López Aguilar, M.F. Webster, H.R. Tamaddon Jahromi, et al, J. Non-Newtonian Fluid Mech. 222 (2015) 190-208.

[2] H.R. Tamaddon Jahromi, M.F. Webster, P.R. Williams, J. Non-Newton Fluid Mech. 166 (2011) 939-950.

[3] D.G. Hassell, D. Auhl, T.C.B. McLeish, et al, Rheol. Acta 47 (2008) 821-834.

[4] D.G. Hassell, T.D. Lord, L. Scelsi, et al, Rheol. Acta 50 (2011) 675-689.

[5] T. McLeish, R.G. Larson, J. Rheol. 42 (1998) 81-110.

[6] N. Clemeur, R.P.G Rutgers, B. Debbaut, Rheol. Acta 42 (2003) 217-231.

[7] W. Wang, X.K. Li, X.H. Han, J. Non-Newton Fluid Mech. 165 (2010) 1480-1493.

[8] W. Wang, X.P. Wang, C.X. Hu, Korea-Aust. Rheol. J. 29 (2014) 365-375.

[9] W. Wang, C.X. Hu, W.W. Li, Mech. Time-Depend. Mater. (2015)

DOI: $10.1007 / \mathrm{s} 11043-015-9287-3$. 